\title{
Gastkommentar zum Beitrag „Frühgeburtenrate in 6 Perinatalzentren in Baden-Württemberg - Potenzial zur Reduktion der Frühgeborenenzahl“": Vorbeugen ist besser als Heilen
}

\author{
Autor \\ Egbert Herting \\ Institut \\ Klinik für Kinder- und Jugendmedizin, Universitätsklinikum \\ Schleswig-Holstein, Campus Lübeck \\ Bibliografie \\ DOI https://doi.org/10.1055/a-0744-1513 \\ Z Geburtsh Neonatol 2018; 222: 208-209 \\ (c) Georg Thieme Verlag KG Stuttgart · New York \\ ISSN 0948-2393
}

\author{
Korrespondenzadresse \\ Prof. Dr. med. Egbert Herting \\ Klinik für Kinder- und Jugendmedizin \\ Universitätsklinikum Schleswig-Holstein, Campus Lübeck \\ Ratzeburger Allee 160 \\ 23538 Lübeck \\ Egbert.Herting@uksh.de
}

Frühgeburtlichkeit hat sich zur häufigsten Todesursache bei jungen Säuglingen weltweit entwickelt. Die Möglichkeiten, durch medizinische Maßnahmen die Frühgeborenenrate zu senken, sind aber begrenzt und vielfach wenig evidenzbasiert [1]. U.a. diesem Sachverhalt ist es auch geschuldet, dass die deutsche AWMF-Leitlinie „Prävention und Therapie der Frühgeburt“ eine „schwierige Geburt“ ist, die sich schon über einige Jahre erstreckt, aber nun hoffentlich bald „das Licht der Welt“ erblicken wird.

Auch wenn sich durch die in der Literatur beschriebenen Maßnahmen eigentlich die Frühgeborenenrate senken lassen sollte [2], so scheinen derzeit gegenläufige gesellschaftliche Trends (u. a. höheres Alter der Schwangeren, Inanspruchnahme der Reproduktionsmedizin, Zunahme von Mehrlingsgeburten) de facto dafür zu sorgen, dass der relative Anteil der Frühgeburten trotz aller Anstrengungen in Deutschland nicht abfällt.

Ja, Frühgeburt vermeiden („Vorbeugen“) ist besser als Frühgeborene behandeln („Heilen“) - aber wie? Das Thema Frühgeburtlichkeit verdeutlicht, dass Erfolge nur mit einer engen Zusammenarbeit von u. a. Geburtshilfe und Neonatologie im Sinne einer echten Perinatalmedizin erzielt werden können. Die von Trotter und Mitarbeitern aufgeworfene Frage, wie gut sind eigentlich einzelne Zentren in der Verhinderung der Frühgeburtlichkeit, ist höchst relevant. Der Beitrag stößt aber, wie ja auch in der Diskussion selber angemerkt wird, an klare methodische Grenzen. Ähnliche Versuche wurden bereits auch in anderen regionalen Neonatalerhebungen unternommen, zeigen dann aber doch relativ deutlich, dass die Eltern selber (wohl auch z.T. den Ratschlägen von niedergelassenen Kolleginnen und Kollegen folgend), bei Hochrisikoschwangerschaften nicht mehr einfach das nächstgelegene Perinatalzentrum ansteuern, sondern durchaus auch bereit sind, weitere Wege für eine optimale Versorgung in Kauf zu nehmen. Man muss daher davon ausgehen, dass Schwangerschaftsrisiken (u. a. demografi- sche und soziale Einflussfaktoren) nicht gleich zwischen verschiedenen Zentren verteilt sind und die unterschiedlichen Frühgeborenenraten von daher nicht nur die Fähigkeiten des Behandlerteams widerspiegeln.

Die Arbeit unterstreicht deutlich die Notwendigkeit der Forderung der Fachgesellschaften und auch der Bundesfachgruppe Perinatalmedizin beim IQTIG, dass die Qualitätssicherungsdaten der neonatologischen und geburtshilflichen Erhebung dringend im Sinne eines echten perinatalmedizinischen Ansatzes zusammenzuführen sind. Dann könnten z. B. auch ohne die „methodischen Umwege“ dieser Studie solche und ähnliche Fragestellungen untersuchbar werden, und auch das Problem, nur $70 \%$ der OutcomeDaten der Neugeborenen zur Verfügung zu haben, wie in der aktuellen Arbeit, könnte umgangen werden.

Um aus den in der Untersuchung beobachteten Unterschieden lernen zu können und um besser zu werden, bräuchte es aber klare Evidenz, welche strukuturellen, personellen, präventiven und therapeutischen Maßnahmen wirklich erfolgreich Frühgeburtlichkeit verhindern können. Auf dieses Dilemma weist auch der geburtshilfliche Gastkommentar zu dem Beitrag (vgl. oben) ausdrücklich hin.

2005 erschienen zeitgleich ein Strukturpapier der Fachgesellschaften [3] und der erste Beschluss des gemeinsamen Bundesausschusses (G-BA). Die Einführung und Scharfschaltung des DRG-Systems genau zu dieser Zeit der Bedrohung des Bestandes von vielen Kinderkliniken in der Fläche [4] führte zu einer Schärfe der Diskussion zwischen „großen und kleinen Neonatologien“, die damals fast die Gesellschaft für Neonatologie und Pädiatrische Intensivmedizin (GNPI) zu sprengen drohte. Das Ziel der G-BA-Beschlüsse, zunächst durch Mindestmengen und dann durch Verschärfung von Strukturvoraussetzungen eine Zentralisierung der perinatalmedizinischen Versorgung zu erreichen, wurde in den letzten 10 Jahren 
nicht umgesetzt. Erfreulicherweise bleibt aber festzustellen, dass bestimmte Maßnahmen, wie eine obligate Infektionssurveillance und die Festschreibung von Personalvorgaben für die Intensivpflege, immerhin dazu geführt haben, dass z. B. die Rate an nosokomialen Infektionen in der Neonatologie in Deutschland gesunken ist. Auch die Festschreibung der Notwendigkeit einer psychosozialen Betreuung für die Familien von Frühgeborenen und die damit festgelegte Finanzierung im DRG-System ist als klarer Gewinn zu bezeichnen.

Bei allem Ärger über endlose Listen, die für den G-BA auszufüllen sind, hat das Augenmerk des G-BA auf die Neonatologie und die Versorgung kleiner Frühgeborener und Ihrer Familien der Sache sicherlich eher genutzt als geschadet. Die meisten Frühgeborenenintensivstationen verfügen heute über eine deutlich verbesserte (v. a. pflegerische) Ausstattung, die ohne den Druck des G-BA auf Kostenträger, Verwaltungen und Vorstände so sicher nicht zustande gekommen wäre.

Betrachtet man die Tatsache, dass Deutschland in Perinatalstatistiken einen guten, aber keinen Spitzenplatz einnimmt und dabei pro Kopf der Bevölkerung relativ viel Geld für Gesundheitsleistungen ausgibt und bezogen auf die Neugeborenenversorgung die vierfache Anzahl (Schweden) und die doppelte Anzahl (Frankreich) an neonatologischen Intensivpflegeplätzen pro Kopf der Bevölkerung vorhält (bei deutlich höheren Geburtenzahlen pro Frau in diesen Ländern), wird der politische Druck, was die Zentralisierung angeht (sowohl der Geburtshilfe als auch der Neonatologie), bestehen bleiben. Der G-BA beschäftigt sich daher derzeit erneut mit dem Thema Mindestmengen in der Neonatologie.

Der Gastkommentar aus gesundheitsökonomischer Sicht (vgl. unten) unterstreicht den Aspekt, dass eine Zentralisierung, die „eher vom freien Spiel der Kräfte“ getrieben wird (beeinflusst von Struktur- und Personalvorgaben des G-BA und einem zunehmen- den Kostendruck einerseits und in immer größerem Umfang auch einem Personalmangel andererseits) wohl kaum zu optimalen $\mathrm{Er}$ gebnissen führen kann. Hinweise dafür, dass es durch „ökonomische Fehlanreize“ zu einem Anstieg der iatrogen bedingten Frühgeborenenrate in Deutschland gekommen wäre, gibt es erfreulicherweise nicht [5].

Der vorliegende Beitrag liefert von daher sicher Denkanstöße, kann aber keine fertigen Lösungen bieten. Entscheidend gerade in Zeiten des Fachkräftemangels dürfte es in Zukunft sein, regional zu guten gestuften Versorgungsstrukturen in der Perinatalmedizin zu kommen getreu dem Motto: „Groß gemeinsam mit Klein und nicht Groß gegen Klein“.

\section{Interessenkonflikt}

Die Autoren geben an, dass kein Interessenkonflikt besteht.

\section{Literatur}

[1] World Health Organization. Preterm birth, 19 February 2018 http:// www.who.int/news-room/fact-sheets/detail/preterm-birth

[2] Goldenberg RL, Rouse D]. Prevention of premature birth. New Engl ] Med 1998; 339: 313-320

[3] Bauer K, Vetter K, Groneck P et al. Empfehlungen für die strukturellen Voraussetzungen der perinatologischen Versorgung in Deutschland. Z Geburtsh Neonatol 2006; 210: 19-24

[4] Weyersberg A, Roth B, Woopen C. Pädiatrie: Folgen der Ökonomisierung. Dtsch Arztebl 2018; 115: A-382

[5] Herting E. Finanzierung: Zu Handlangern degradiert. Dtsch Arztebl 2011; 108: A-1122 